\title{
Andreev levels in a single-channel conductor
}

Titov, M.; Mortensen, Asger; Schomerus, H.; Beenakker, C.W.J.

\section{Published in:}

Physical Review B

Link to article, DOI:

10.1103/PhysRevB.64.134206

Publication date:

2001

Document Version

Publisher's PDF, also known as Version of record

Link back to DTU Orbit

Citation (APA):

Titov, M., Mortensen, A., Schomerus, H., \& Beenakker, C. W. J. (2001). Andreev levels in a single-channel conductor. Physical Review B, 64(13), 134206. https://doi.org/10.1103/PhysRevB.64.134206

\section{General rights}

Copyright and moral rights for the publications made accessible in the public portal are retained by the authors and/or other copyright owners and it is a condition of accessing publications that users recognise and abide by the legal requirements associated with these rights.

- Users may download and print one copy of any publication from the public portal for the purpose of private study or research.

- You may not further distribute the material or use it for any profit-making activity or commercial gain

- You may freely distribute the URL identifying the publication in the public portal

If you believe that this document breaches copyright please contact us providing details, and we will remove access to the work immediately and investigate your claim. 


\title{
Andreev levels in a single-channel conductor
}

\author{
M. Titov, ${ }^{1}$ N. A. Mortensen, ${ }^{1,2}$ H. Schomerus, ${ }^{1,3}$ and C. W. J. Beenakker ${ }^{1}$ \\ ${ }^{1}$ Instituut-Lorentz, Universiteit Leiden, P. O. Box 9506, 2300 RA Leiden, The Netherlands \\ ${ }^{2}$ Mikroelektronik Centret, Technical University of Denmark, Orsteds Plads 345 E, 2800 Lyngby, Denmark \\ ${ }^{3}$ Max-Planck-Institut für Physik komplexer Systeme, Nöthnitzer Straße 38, 01187 Dresden, Germany
}

(Received 21 March 2001; published 11 September 2001)

\begin{abstract}
We calculate the subgap density of states of a disordered single-channel normal metal connected to a superconductor at one end (normal-metal-superconductor junction) or at both ends [superconductor-normalmetal-superconductor (SNS) junction]. The probability distribution of the energy of a bound state (Andreev level) is broadened by disorder. In the SNS case the twofold degeneracy of the Andreev levels is removed by disorder leading to a splitting in addition to the broadening. The distribution of the splitting is given precisely by Wigner's surmise from random-matrix theory. For strong disorder the mean density of states is largely unaffected by the proximity to the superconductor, because of localization, except in a narrow energy region near the Fermi level, where the density of states is suppressed with a log-normal tail.
\end{abstract}

DOI: $10.1103 /$ PhysRevB.64.134206

PACS number(s): 74.80.Fp, 72.15.Rn, 73.63.Rt

\section{INTRODUCTION}

Several recent works have identified and studied deviations from mean-field theory in the subgap density of states of a normal metal in contact with a superconductor. ${ }^{1-4}$ The excitation spectrum below the gap of the bulk superconductor consists of a coherent superposition of electron and hole excitations, coupled by Andreev reflection ${ }^{5}$ at the normal-metal-superconductor (NS) interface. The energy of these Andreev levels fluctuates from sample to sample, but such mesoscopic fluctuations are ignored in mean-field theory. Because of these fluctuations, the ensemble averaged density of states $\langle\nu(\varepsilon)\rangle$ acquires a tail that extends below the mean-field gap, vanishing only at the Fermi level (zero excitation energy $\varepsilon$ ). The fluctuations become particularly large if the size of the normal metal is greater than the localization length.

The purpose of this paper is to analyze an extreme case of complete breakdown of mean-field theory, which is still sufficiently simple that it can be solved exactly. This is the case of single-mode conduction through a disordered normalmetal wire attached to a superconductor. The localization length in this geometry is equal to the elastic mean-free path $l$, so that the wire crosses over with increasing length $L$ from the ballistic regime directly into the localized regimewithout an intermediate diffusive regime. Perturbation theory is possible in the quasiballistic regime $l \gg L$, but for $l<L$ an essentially nonperturbative approach is required. We will use an approach based on a scaling equation (also known as invariant embedding) that has proved its use before in different contexts. ${ }^{6-9}$

We will contrast the quasiballistic and localized regimes, as well as the two geometries with a single superconducting contact (NS junction) or with two superconducting contacts at both ends of the normal metal wire [superconductornormal-metal-superconductor (SNS) junction]. If we assume that the two superconductors have the same phase, so that there is no supercurrent flowing through the normal metal, then the Andreev levels of the SNS junction are doubly degenerate in the absence of disorder. This degeneracy is broken by disorder. We find that for weak disorder the probabil- ity distribution of the splitting is given precisely by Wigner's surmise from random-matrix theory. ${ }^{10}$ (The spectra of chaotic systems have spacings described by Gaudin's distribution, which is close to, but not identical with Wigner's surmise. ${ }^{10}$ )

In the localized regime the fluctuations of the Andreev levels become greater than their spacing, and they can no longer be distinguished in the mean density of states, which decreases smoothly to zero on approaching the Fermi level. The energy scale for this soft gap is exponentially small because of localization, given by $\varepsilon_{g}=\left(\hbar v_{F} / l\right) e^{-L / l}$. The decay of $\langle\nu(\varepsilon)\rangle$ for $\varepsilon \ll \varepsilon_{g}$ has a log-normal form $\propto \operatorname{exp[}$ $\left.-(l / 4 L) \ln ^{2}\left(\varepsilon / \varepsilon_{g}\right)\right]$. Such log-normal tails are characteristic of rare fluctuations in the localized regime ${ }^{11}$ and have appeared recently in the context of the superconductor proximity effect. $^{4}$

\section{QUASIBALLISTIC REGIME}

\section{A. NS junction}

The NS junction consists of a piece of normal metal of length $L$ connected at one end to a superconductor and closed at the other end [see Fig. 1(a)]. The width of the normal metal is of the order of the Fermi wavelength $\lambda_{F}$, such that there is a single propagating mode at the Fermi energy $E_{F}$. We assume an ideal junction, without any tunnel barrier and with $E_{F}$ much greater than the superconducting

(a)

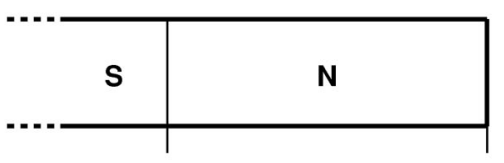

(b)

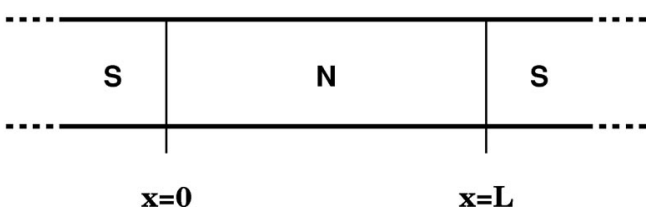

FIG. 1. Geometry of the NS and SNS junctions. 
gap $\Delta_{0}$. An electron incident on the superconductor with energy $\varepsilon<\Delta_{0}$ above the Fermi level is then Andreev reflected as a hole at energy $\varepsilon$ below the Fermi level, with the phase shift

$$
\phi_{A}=-\arccos \left(\varepsilon / \Delta_{0}\right), \quad-\pi / 2<\phi_{A}<0 .
$$

We wish to know at which $\varepsilon$ a bound state (Andreev level) will form in the normal metal.

The electron and hole components of the wave function $\psi(x)=[u(x), v(x)]$ satisfy the Bogoliubov-de Gennes $(\mathrm{BdG})$ equation ${ }^{12}$

$$
\left(\begin{array}{cc}
\mathcal{H}_{0} & \Delta \\
\Delta^{*} & -\mathcal{H}_{0}^{*}
\end{array}\right) \psi=\varepsilon \psi,
$$

where $\mathcal{H}_{0}=-\left(\hbar^{2} / 2 m\right) \partial^{2} / \partial x^{2}+V(x)$ is the Hamiltonian of the normal metal (with disorder potential $V$ ) and $\Delta(x)$ $=\Delta_{0} \theta(-x)$ is the superconducting gap (which vanishes in the normal-metal region $x>0$ ). For narrow junctions (width much less than the superconducting coherence length $\xi_{0}$ $\left.=\hbar v_{F} / \Delta_{0}\right)$ the depletion of $\Delta(x)$ on the superconducting side may be neglected, hence the step function $\theta(-x)$. At the closed end $x=L$ of the normal metal we impose the boundary condition $\psi(L)=0$.

In this section we address the quasiballistic regime of mean-free path $l \gg L$. We can then treat $V$ as a small perturbation on the ballistic bound states

$$
\begin{gathered}
\psi(x)=\frac{1}{\sqrt{Z}}\left(\begin{array}{c}
\sin \left[\left(k_{F}+k\right)(x-L)\right] \\
\sin \left[\left(k_{F}-k\right)(x-L)-\pi n\right]
\end{array}\right), \quad 0<x<L, \\
\psi(x)=\frac{1}{\sqrt{Z}\left(\begin{array}{c}
\sin \left[k_{F} x-\left(k_{F}+k\right) L\right] \\
\sin \left[k_{F} x-\left(k_{F}-k\right) L-\pi n\right]
\end{array}\right)} \\
\quad \times \exp \left(-\frac{x}{\xi_{0}} \sin \phi_{A}\right), \quad x<0 .
\end{gathered}
$$

The normalization constant is $Z=L-\frac{1}{2} \xi_{0} / \sin \phi_{A}$ for $k_{F} L$ $\gg 1$. (We denote $k_{F}=m v_{F} / \hbar=2 \pi / \lambda_{F}$.) The wave number $k=\varepsilon / \hbar v_{F}$ should satisfy the quantization condition

$$
2 k L+\phi_{A}=\pi n, \quad n=0,1,2, \ldots
$$

The total number of Andreev levels within the gap is $2 L / \pi \xi_{0}$ for $L \gg \xi_{0}$. (There remains one level if $L \ll \xi_{0}$.)

To first order in $V$ the energy level is shifted by the matrix element

$$
\delta \varepsilon=\int_{0}^{L} d x V(x)\left[u(x)^{2}-v(x)^{2}\right]
$$

We assume a potential with a short-range correlation, expressed by

$$
\langle V(x)\rangle=0, \quad\left\langle V(x) V\left(x^{\prime}\right)\right\rangle=\frac{\hbar^{2} v_{F}^{2}}{l} \delta\left(x-x^{\prime}\right),
$$

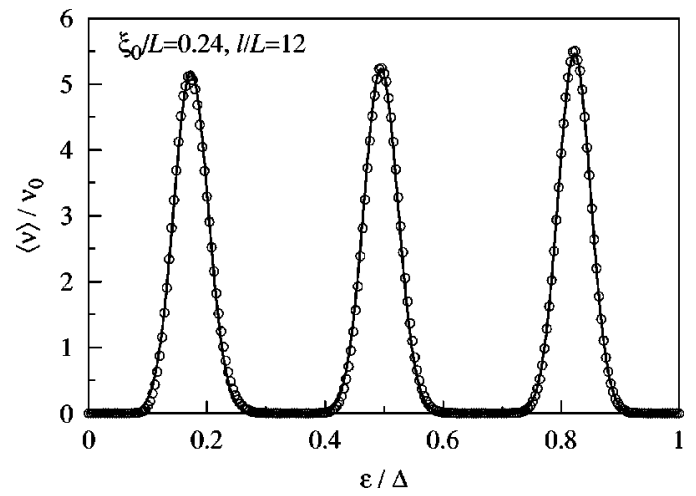

FIG. 2. Mean density of states (in units of $\nu_{0}=2 L / \pi \hbar v_{F}$ ) of a quasiballistic NS junction. The Gaussian with variance given by Eq. (7) (solid curves) is compared to the numerical solution of the BdG equation (data points).

where $\langle\cdots\rangle$ stands for the disorder average. It follows that the distribution of an Andreev level around its ballistic value is a Gaussian with zero mean, $\langle\delta \varepsilon\rangle=0$, and variance

$$
\left\langle\delta \varepsilon^{2}\right\rangle=\frac{\hbar^{2} v_{F}^{2}\left(2 L+\xi_{0} \sin \phi_{A}\right)}{2 l\left(2 L-\xi_{0} / \sin \phi_{A}\right)^{2}} .
$$

By way of illustration, we show in Fig. 2 the mean density of states of an NS junction containing three Andreev levels $\left(\xi_{0} / L=0.24\right)$ with mean-free path $l=12 L$. The Gaussian given by Eq. (7) agrees very well with the numerical solution of the BdG equation (data points).

We briefly explain the numerical method. The BdG equation is solved numerically on a one-dimensional grid (lattice constant $a$ ) by replacing the Laplacian by finite differences and truncating the Hamiltonian matrix in the superconducting region, where the wave function is evanescent for energies in the superconducting gap. The resulting tight-binding model has nearest-neighbor coupling $\gamma=\hbar^{2} / 2 m a^{2}$ (bandwidth $4 \gamma$ ). We set $E_{F}=\gamma$ and $\Delta_{0}=0.1 \gamma$, corresponding to $\lambda_{\mathrm{F}}=6 a$ and $\xi_{0}=10 \sqrt{3} a$. The disorder is modeled by a random on-site potential which is uniformly distributed in the interval $(-W, W)$. The mean-free path from the Born approximation, $l=3 E_{F}\left(4 \gamma-E_{F}\right) a / W^{2}$, was found to fit well to the prediction of one-dimensional scaling theory for the mean inverse transmission probability, $\left\langle T^{-1}\right\rangle=\frac{1}{2}[1$ $+\exp (2 L / l)]$, in the complete range from the quasiballistic to the localized regime. (The localization length $\xi$ is related to the mean-free path by $\xi=2 l$, cf. Ref. 6.) This allows for a parameter-free comparison of the analytical and numerical results for the ensemble-averaged density of states.

\section{B. SNS junction}

The quasiballistic regime in an SNS junction [Fig. 1(b)] is qualitatively different from the NS case of the preceding section. The reason is the double degeneracy of the unperturbed Andreev levels. This degeneracy exists if the phase of the order parameter in the two superconductors is the same, which is what we assume in this paper. Let us examine the splitting of the Andreev levels by the disorder potential. 
The SNS junction has energy gap

$$
\Delta(x)=\Delta_{0} \theta(-x)+\Delta_{0} \theta(x-L) .
$$

The quantization condition reads

$$
k L+\phi_{A}=n \pi, \quad n=0,1,2, \ldots
$$

There are $L / \pi \xi_{0}$ Andreev levels (for $L \gg \xi_{0}$ ), each level being doubly degenerate. We choose the two independent eigenfunctions $\psi_{ \pm}(x)$ such that they carry zero current. They are given by

$$
\begin{gathered}
\psi_{+}(x)=\frac{1}{\sqrt{Z^{\prime}}}\left(\begin{array}{c}
\cos \left(k_{F} x\right) \\
\cos \left(k_{F} x-\phi_{A}\right)
\end{array}\right) \exp \left(-\frac{x}{\xi_{0}} \sin \phi_{A}\right), \quad x<0, \\
\psi_{+}(x)=\frac{1}{\sqrt{Z^{\prime}}}\left(\begin{array}{c}
\cos \left[\left(k_{F}+k\right) x\right] \\
\cos \left[\left(k_{F}-k\right) x-\phi_{A}\right]
\end{array}\right), \quad 0<x<L, \\
\psi_{+}(x)=\frac{1}{\sqrt{Z^{\prime}}}\left(\begin{array}{c}
\cos \left(k_{F} x+k L\right) \\
\cos \left(k_{F} x+\pi n\right)
\end{array}\right) \exp \left(\frac{x-L}{\xi_{0}} \sin \phi_{A}\right), \quad x>L,
\end{gathered}
$$

and $\psi_{-}(x)$ is obtained by replacing cosine by sine. The normalization constant is now $Z^{\prime}=L-\xi_{0} / \sin \phi_{A}$.

To first order in $V$ the levels are splitted symmetrically around the ballistic value, by an amount $\pm \frac{1}{2} s$. The basis (10) is chosen in such a way that the off-diagonal elements of the perturbation vanish. The shift of each level can then be calculated from Eq. (5) using the corresponding eigenfunction. We again calculate the probability distribution $P(s)$ of the level splitting using Eq. (6). The result is

$$
P(s)=\frac{\pi s}{2\langle s\rangle^{2}} \exp \left(-\frac{\pi s^{2}}{4\langle s\rangle^{2}}\right)
$$

with average splitting

$$
\langle s\rangle=\Delta \sqrt{\frac{\pi}{2 l}} \frac{\xi_{0} \sqrt{L+\xi_{0} \sin \phi_{A}}}{L-\xi_{0} / \sin \phi_{A}} .
$$

We recognize Eq. (11) as Wigner's surmise of randommatrix theory. ${ }^{10}$

In Fig. 3 we compare Eq. (11) with numerical data. The agreement is excellent for a range of mean-free paths in the quasiballistic regime. The mean position of the splitted levels fluctuates only to higher orders in $L / l$. This makes it possible to resolve the splitting in the mean density of states (see inset in Fig. 3).

\section{LOCALIZED REGIME}

\section{A. NS junction}

In order to go beyond the quasiballistic regime into the localized regime $L \gg l$ we write the quantization condition for the Andreev levels in an NS junction in the form

$$
r(\varepsilon) r(-\varepsilon) * e^{2 i \phi_{A}=1,}
$$

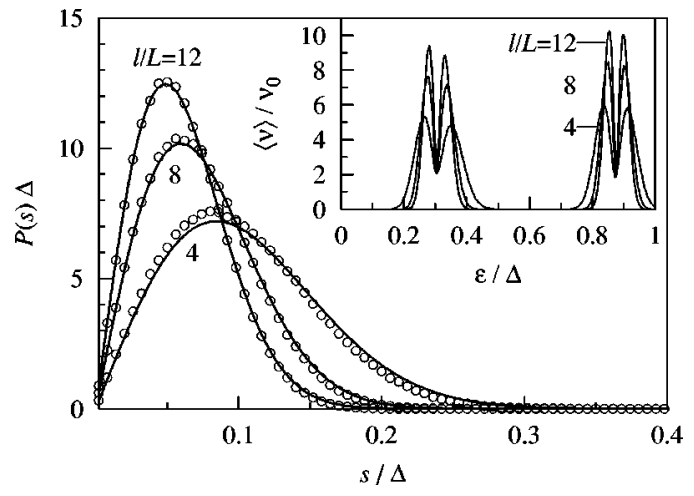

FIG. 3. Distribution of the splitting $s$ of the first pair of Andreev levels in an SNS junction with $\xi_{0} / L=0.24$. The solid curves are our theoretical expectation from Eq. (11), the data points result from the numerical solution of the BdG equation. The inset shows the numerical data for the mean density of states.

where $r(\varepsilon)=e^{i \phi(\varepsilon)}$ is the reflection amplitude of the disordered normal metal. [The hole has reflection amplitude $\left.r^{*}(-\varepsilon).\right]$ In terms of the phase shifts we have

$$
\Phi(\varepsilon) \equiv \frac{\phi(\varepsilon)-\phi(-\varepsilon)}{2}+\phi_{A}(\varepsilon)=\pi n, \quad n=0,1, \ldots
$$

The density of states $\nu(\varepsilon)=\Sigma_{n} \delta\left(\varepsilon-\varepsilon_{n}\right)$ is related to the scattering phase shifts by ${ }^{13}$

$$
\nu(\varepsilon)=-\frac{1}{\pi} \frac{d}{d \varepsilon} \operatorname{Im} \ln \sin \Phi\left(\varepsilon+i 0^{+}\right),
$$

where $0^{+}$denotes a positive infinitesimal. The imaginary part of the logarithm jumps by $\pi$ whenever $\sin \Phi(\varepsilon)$ changes sign, hence it counts the number of levels below $\varepsilon$. The derivative with respect to $\varepsilon$ then gives the density of states. It is convenient to write Eq. (15) as a Taylor series,

$$
\nu(\varepsilon)=\frac{1}{\pi} \frac{d}{d \varepsilon}\left(\Phi+\operatorname{Im} \sum_{m=1}^{\infty} \frac{1}{m} e^{2 i m \Phi}\right),
$$

which converges because $\Phi\left(\varepsilon+i 0^{+}\right)$is equivalent to $\Phi(\varepsilon)+i 0^{+}$.

We seek the disorder-averaged density of states $\langle\nu(\varepsilon)\rangle$. One way to proceed is by means of the Berezinskii technique. ${ }^{14,15}$ An alternative way, that we will follow here, is to start from the scaling equation ${ }^{7,8}$ for the probability distribution $P\left(\phi_{N}\right)$ of the phase shift $\phi_{N}=\frac{1}{2}[\phi(\varepsilon)$ $-\phi(-\varepsilon)]$. This equation has the form

$$
\frac{\partial P}{\partial L}=\frac{\partial}{\partial \phi_{N}}\left(-\frac{2 \varepsilon}{\hbar v_{F}}+\frac{1}{l} \frac{\partial}{\partial \phi_{N}} \sin ^{2} \phi_{N}\right) P .
$$

The initial condition is $\lim _{L \rightarrow 0} P\left(\phi_{N}\right)=\delta\left(\phi_{N}\right)$.

The first moment satisfies $\partial\left\langle\phi_{N}\right\rangle / \partial L=2 \varepsilon / \hbar v_{F}$, hence

$$
\left\langle\phi_{N}\right\rangle=\frac{2 \varepsilon L}{\hbar v_{F}} \text {. }
$$

Multiplication of Eq. (17) by $\exp \left(2 i m \phi_{N}\right)$ and integration over $\phi_{N}$ from 0 to $\pi$ yields a set of recursive differential equations ${ }^{14}$ for the moments $R_{m}=\left\langle e^{2 i m \phi_{N}}\right\rangle$, 


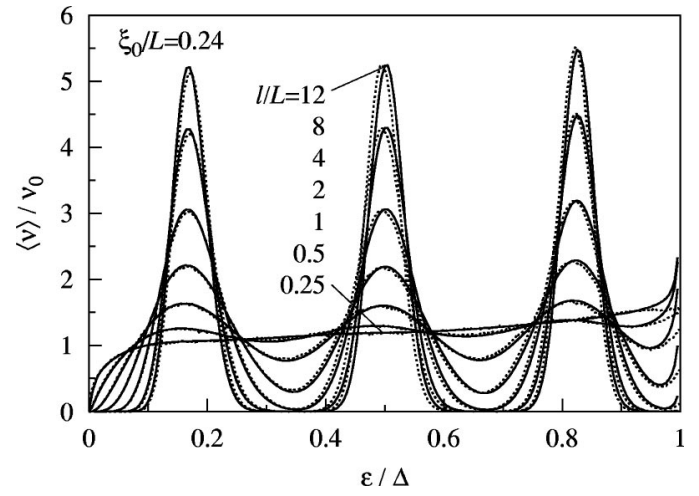

FIG. 4. Mean density of states of an NS junction from the quasiballistic into the localized regime. The solid curves have been computed from Eqs. (16) and (19). The dashed curves are a numerical simulation of random disorder in the BdG equation.

$$
\frac{\partial R_{m}}{\partial L}=\frac{m^{2}}{l}\left(R_{m+1}+R_{m-1}-2 R_{m}\right)+\frac{4 i \varepsilon}{\hbar v_{F}} m R_{m},
$$

with the initial condition $R_{m}(0)=1$. We solve this set of equations by truncating the vector $\left(R_{1}, R_{2}, \ldots R_{M}\right)$ at a sufficiently large value of $M \approx 400$ and diagonalizing the corresponding tridiagonal matrix. From Eq. (16) we then find the mean density of states.

The result is shown in Fig. 4 for $\xi_{0} / L=0.24$ and ratios $l / L$ ranging from the quasiballistic regime to the localized regime. Agreement with the numerical solution of the $\mathrm{BdG}$ equation is excellent over the whole range.

In the localized regime $L \gg l$ the individual Andreev levels can no longer be distinguished in the mean density of states, because the broadening of the levels becomes greater than the spacing. In this regime we distinguish two energy ranges, $\varepsilon \gg \varepsilon_{g}$ and $\varepsilon \ll \varepsilon_{g}$, where $\varepsilon_{g}=\left(\hbar v_{F} / l\right) e^{-L / l}$.

For energies higher than $\varepsilon_{g}$ we may use the $L \rightarrow \infty$ limit of the distribution $P\left(\phi_{N}\right)$, obtained by setting the left-hand side of Eq. (17) equal to zero. The resulting moments are

$$
\left.\lim _{L \rightarrow \infty} R_{m}=\int_{0}^{\infty} d \sigma e^{-\sigma\left(\frac{\sigma}{\sigma-i \omega}\right.}\right)^{m}, \quad \omega=\frac{4 \varepsilon l}{\hbar v_{F}} .
$$

We then calculate the mean density of states from Eq. (16), with the result

$$
\begin{gathered}
\langle\nu(\varepsilon)\rangle=\frac{2 L}{\pi \hbar v_{F}}+\frac{1}{\pi \sqrt{\Delta_{0}^{2}-\varepsilon^{2}}}+f(\varepsilon), \quad \varepsilon \gg \varepsilon_{g}, \\
f(\varepsilon)=\frac{\partial}{\partial \varepsilon} \operatorname{Im} \int_{0}^{\infty} \frac{d \sigma}{\pi}\left[\frac{e^{-\sigma}}{\sigma-i \omega}-\frac{e^{-\sigma}\left(1-e^{2 i \phi_{A}}\right)}{\sigma\left(1-e^{2 i \phi_{A}}\right)-i \omega}\right] .
\end{gathered}
$$

The first term on the right-hand side of Eq. (21) is the energy independent density of states $\nu_{0}$ in an isolated normal metal. The main effect of the superconductor for $\varepsilon \gg \varepsilon_{g}$ is an enhancement of the density of states close to the gap $\Delta_{0}$ of the bulk superconductor (second term). The third term is negative for sufficiently small $\varepsilon$ and is a precursor of the soft gap near the Fermi level. For $\xi_{0} \ll l$ and $\varepsilon \ll \hbar v_{F} / l$ the reduction term $f(\varepsilon)$ can be simplified as

$$
f(\varepsilon)=-\frac{2 l}{\pi \hbar v_{F}}\left(\ln \frac{\hbar v_{F}}{8 \varepsilon l}-\gamma\right), \quad \varepsilon_{g} \ll \varepsilon \ll \frac{\hbar v_{F}}{l},
$$

where $\gamma \approx 0.58$ is Euler's constant.

Near the Fermi level, for $\varepsilon \ll \varepsilon_{g}$, the mean density of states vanishes as a result of the proximity to the superconductor. This "soft gap" appears no matter how strongly localized the normal metal is. The coefficients $R_{m}$ may now be treated as analytical functions of the parameter

$$
z=-\frac{4 i \varepsilon l m}{\hbar v_{F}}, \quad R_{m}=R(z) .
$$

Taking the limit $\varepsilon \rightarrow 0$ we deduce from Eq. (17) the partialdifferential equation

$$
l \frac{\partial R}{\partial L}=z^{2} \frac{\partial^{2} R}{\partial z^{2}}-z R
$$

with initial condition $\lim _{L \rightarrow 0} R(z)=1$. This differential equation has been studied before in the theory of one-dimensional localization, ${ }^{16,17}$ but not in connection with the proximity effect. The result for the mean density of states, derived in the Appendix, is given by

$$
\langle\nu(\varepsilon)\rangle=\frac{2 l}{\pi^{3 / 2} \hbar v_{F}} \exp \left[-\frac{l}{4 L} \ln ^{2} \frac{\pi \varepsilon_{g}}{\varepsilon}-\frac{u l}{2 L}\left(\ln \frac{u l}{2 L}-1\right)\right],
$$

where $u=\ln \pi \hbar v_{F} / \varepsilon l=\ln \pi \varepsilon_{g} / \varepsilon+L / l$. The leading logarithmic asymptotic of this expression in the limit $\varepsilon \ll \varepsilon_{g}$ has the log-normal tail

$$
\langle\nu(\varepsilon)\rangle \propto \exp \left[-\frac{l}{4 L} \ln ^{2} \frac{\pi \varepsilon_{g}}{\varepsilon}\right], \quad \varepsilon \ll \varepsilon_{g} .
$$

The same log-normal tail was found in Ref. 4 for a manychannel diffusive conductor. In that case the factor $l / L$ is replaced by the Drude conductance of the normal metal and the energy scale $\varepsilon_{g}$ is replaced by the Thouless energy $\hbar D / L^{2}$ (with $D$ the diffusion constant). In our single-channel localized conductor neither the Drude conductance nor the Thouless energy play a role.

\section{B. SNS junction}

In contrast with the quasiballistic regime, the NS and SNS junctions are similar in the localized regime. (At least for the case of zero current through the SNS junction considered here.) Unfortunately, there exists no simple scaling equation as Eq. (17) that can describe the density of states of the SNS junction. We therefore rely on the numerical solution of the BdG equation. In Fig. 5 we show that the mean density of states of an NS junction of length $L$ is close to that of an SNS junction of length $2 L$. This factor of 2 has an obvious explanation in the ballistic regime [compare Eqs. (4) and (9)], but it is remarkable that it still applies to the localized regime.

\section{CONCLUSION}

In summary, we have calculated the effect of disorder on the spectrum of Andreev levels in single-channel NS and SNS junctions. The nonperturbative effects of localization in 


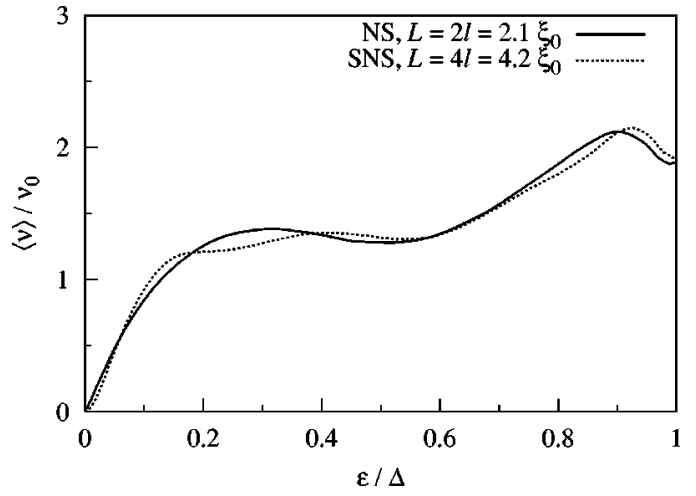

FIG. 5. Numerical calculation of the mean density of states of an NS junction (solid) and SNS junction (dashed) in the nearly localized regime. The length of the SNS junction is twice that of the NS junction. (The weak oscillations are remnants of Andreev levels, that will disappear if $L / l$ is increased further.)

the one-dimensional case can be studied exactly, at least in the NS geometry. Our research is of theoretical interest in view of recent studies of the subgap density of states beyond mean-field theory, ${ }^{1-4}$ but may also be of experimental interest in view of recent progress made in superconductorcarbon-nanotube devices. ${ }^{18,19}$

The results derived in the quasiballistic regime are not restricted to a one-dimensional geometry. Andreev levels of an SNS junction remain doubly degenerate in higher dimensions without disorder, and weak disorder will still induce a splitting distributed according to the Wigner surmise. The subgap density of states in the localized regime has been studied in higher dimensions without disorder in Ref. 4. The log-normal tail is a generic feature of the lowest energies.

\section{ACKNOWLEDGMENTS}

We thank Piet Brouwer for a crucial discussion at the initial stage of this project. This research was supported by the "Nederlandse organisatie voor Wetenschappelijk Onderzoek" (NWO) and by the "Stichting voor Fundamenteel Onderzoek der Materie" (FOM). M.T. and N.A.M. thank the visitors program at the Max-Planck-Institut für Physik komplexer Systeme, Dresden. N.A.M. also acknowledges support by the "Ingeniørvidenskabelig Fond og G.A. Hagemanns Mindefond."

\section{APPENDIX: DERIVATION OF THE LOG-NORMAL TAIL}

The differential operator on the right-hand side of Eq. (25) has eigenfunctions

$$
f_{p}(z)=2 \sqrt{z} K_{p}(2 \sqrt{z})
$$

where $K_{p}(z)$ is the modified Bessel function, such that

$$
\left(z^{2} \frac{\partial^{2}}{\partial z^{2}}-z\right) f_{p}(z)=\frac{p^{2}-1}{4} f_{p}(z) .
$$

The solution to Eq. (25) with the initial condition $\lim _{L \rightarrow 0} R(z)=1$ is

$$
\begin{aligned}
R(z)= & f_{1}(z)+\int_{-\infty}^{\infty} d \nu \frac{\nu \sinh (\pi \nu / 2)}{\pi\left(\nu^{2}+1\right)} f_{i \nu}(z) \\
& \times \exp \left[-\left(\nu^{2}+1\right) L / 4 l\right] .
\end{aligned}
$$

To obtain the density of states of the NS junction it is convenient to define the inverse Laplace transform

$$
F(\lambda)=\frac{1}{2 \pi i} \int_{-i \infty+0^{+}}^{i \infty+0^{+}} \frac{d z}{(4 \lambda)^{2}} R(z) \exp \left(\frac{z}{4 \lambda}\right) .
$$

From Eq. (16) we find for $\varepsilon \ll \varepsilon_{g}$ the mean density of states in terms of the function $F$,

$$
\langle\nu(\varepsilon)\rangle=\frac{4 l}{\pi \hbar v_{F}} F\left(\frac{\varepsilon l}{\pi \hbar v_{F}}\right) .
$$

Our aim is to find the asymptotic form of $F(\lambda)$ in the limit $\lambda \rightarrow 0$. The inverse Laplace transform of the modified Bessel functions in Eq. (A3) can be found in Ref. 20. We obtain

$$
\begin{aligned}
F(\lambda)= & F_{0}(\lambda)-\int_{-\infty}^{\infty} d \nu \lambda^{-(i \nu+1) / 2} \exp \left[-\left(\nu^{2}+1\right) L / 4 l\right] \\
& \times \frac{{ }_{1} F_{1}\left(\frac{3}{2}+\frac{i \nu}{2}, 1+i \nu,-4 \lambda\right)}{2 \sqrt{\pi}(1-i \nu) \Gamma(i \nu / 2)},
\end{aligned}
$$

where $F_{0}(\lambda)=\exp (-4 \lambda)$. The integrand has a single pole $\nu=-i$ in the lower half of the complex plane and the residue from this pole cancels the term $F_{0}$. Let us shift the contour by the transformation $\nu \rightarrow \nu-(i l / L) \ln (1 / \lambda)$ and consider the limit $\lambda \ll e^{-L / l}$. In this limit the contour is shifted through the pole so that the term $F_{0}$ is canceled. Moreover, the hypergeometric function ${ }_{1} F_{1}$ can be replaced by unit in this limit. Thus, we end up with the integral

$$
\begin{aligned}
F(\lambda)= & \frac{1}{2 \sqrt{\pi}} \exp \left[-\frac{l}{4 L}\left(\ln \frac{1}{\lambda}-\frac{L}{l}\right)^{2}\right] \int_{-\infty}^{\infty} d \nu e^{-\nu^{2} L / 4 l} \\
& \times\left[\left(i \nu-1-\frac{l}{L} \ln \lambda\right) \Gamma\left(\frac{i \nu}{2}-\frac{l}{2 L} \ln \lambda\right)\right]^{-1} .
\end{aligned}
$$

The asymptotic form of this integral in the limit $\lambda \ll e^{-L / l}$ can be found by evaluation of the expression in square brackets in the point $\nu=0$ and calculation of the Gaussian integral. Using the asymptotic formula for the Euler gamma function one obtains the mean density of states given in Eq. (26).
${ }^{1}$ I. S. Beloborodov, B. N. Narozhny, and I. L. Aleiner, Phys. Rev. Lett. 85, 816 (2000).

${ }^{2}$ A. Lamacraft and B. D. Simons, Phys. Rev. Lett. 85, 4783 (2000); Phys. Rev. B 64, 014514 (2001).
${ }^{3}$ M. G. Vavilov, P. W. Brouwer, V. Ambegaokar, and C. W. J. Beenakker, Phys. Rev. Lett. 86, 874 (2001).

${ }^{4}$ P. M. Ostrovsky, M. A. Skvortsov, and M. V. Feigel'man, Phys. Rev. Lett. 87, 027002 (2001). 
${ }^{5}$ A. F. Andreev, Zh. Eksp. Teor. Fiz. 46, 1823 (1964) [Sov. Phys. JETP 19, 1228 (1964)].

${ }^{6}$ C. W. J. Beenakker, Rev. Mod. Phys. 69, 731 (1997).

${ }^{7}$ B. White, P. Sheng, Z. Q. Zhang, and G. Papanicolaou, Phys. Rev. Lett. 59, 1918 (1987).

${ }^{8}$ M. Titov and C. W. J. Beenakker, Phys. Rev. Lett. 85, 3388 (2000).

${ }^{9}$ M. Titov, P. W. Brouwer, A. Furusaki, and C. Mudry, Phys. Rev. B 63, 235318 (2001).

${ }^{10}$ M. L. Mehta, Random Matrices (Academic, New York, 1991).

${ }^{11}$ A. D. Mirlin, Phys. Rep. 326, 259 (2000).

${ }^{12}$ P. G. de Gennes, Superconductivity of Metals and Alloys (Benjamin, New York, 1966).

${ }^{13}$ E. Doron and U. Smilansky, Phys. Rev. Lett. 68, 1255 (1992).
${ }^{14}$ V. L. Berezinskii, Zh. Eksp. Teor. Fiz. 65, 1251 (1973) [Sov. Phys. JETP 38, 620 (1974)].

${ }^{15}$ V. L. Berezinskii and L. P. Gor'kov, Zh. Eksp. Teor. Fiz. 77, 2498 (1979) [Sov. Phys. JETP 50, 1209 (1979)].

${ }^{16}$ B. L. Altshuler and V. N. Prigodin, Zh. Eksp. Teor. Fiz. 95, 348 (1989) [Sov. Phys. JETP 68, 198 (1989)].

${ }^{17}$ Y. V. Fyodorov and A. D. Mirlin, Int. J. Mod. Phys. B 8, 3795 (1994).

${ }^{18}$ A. F. Morpurgo, J. Kong, C. M. Marcus, and H. Dai, Science 286, 263 (1999).

${ }^{19}$ Y. Wei, J. Wang, H. Guo, H. Mehrez, and C. Roland, Phys. Rev. B 63, 195412 (2001).

${ }^{20}$ A. P. Prudnikov, Yu. A. Brychkov, and O. I. Marichev, Integrals and Series (Gordon and Breach, Amsterdam, 1986). 